\title{
Identification of the hemodynamic significant patent ductus arteriosus in preterm infants
}

\author{
Xiaomin Guo ${ }^{1 *}$, Yong $\mathrm{Ji}^{1}$, Ruirui Wang ${ }^{1}$, Jinzhou Pan ${ }^{1}$ \\ ${ }^{1}$ Department of Pediatrics Women \& Children's Hospital, Taiyuan, Shanxi, China, 030013 \\ *Corresponding Author: Dr. Xiaomin Guo, Department of Pediatrics Women \& Children's Hospital, Taiyuan, Shanxi, China; \\ email address: gxm_001@sina.cn
}

\section{Abstract}

Objective To review the research on the discernible markers of hemodynamic significant patent ductus arteriosus (hsPDA). Date sources We consulted literatures from PubMed in English up to 2020. Study selection Data about sensitivity, specificity and cut-off value and experimental conclusion are from clinical trials and accurate analysis. Result Hemodynamic significant PDA is associated with severe complications and mortality in premature infant. So far, there are still controversies over the treatment of patent ductus arteriosus in preterm infants, mainly relating to identification of hemodynamic significant patent ductus arteriosus (hsPDA) is ununiform. Numbers of scholars used to employ color Doppler markers to evaluate hsPDA in last 4 decades. This review aims to identify hsPDA in preterm infants from three aspects by analysis the literatures, including non-invasive detection index, biological index and clinical index, which providing evidence-based guidance to reduce the occurrence of excessive medical treatment. Conclusion Echocardiographic markers combining with biological and clinical indicators can improve the identification of hsPDA clinically. Despite the definition of hsPDA has been investigated, the diagnosis and treatment remain ambiguous. More comprehensive and meaningful assessment for hsPDA is required.

Keywords: Hemodynamic significant PDA (hsPDA), Preterm infant, Non-invasive markers.

\section{INTRODUCTION}

Patent ductus arteriosus is commonly found in neonates and usually functionally closed in 72 hours after birth, the probability of spontaneous closure of ductus arteriosus is significantly reduced in preterm infants. Studies have shown that the incidence of patent ductus arteriosus is $40 \%-55 \%$ in preterm infants younger than 29 weeks ${ }^{[1]}$, and $50 \%-70 \%$ in extremely low birth weight infant ${ }^{[2]}$. Premature infants are at significant risk for persistent patency of the ductus arteriosus. In the 1980s, researchers have found birth asphyxia and sever respiratory distress syndrome, which have high rates in very low birth weight infants could induce significant shunting of ductus arteriosus [3]. Hemodynamic significant PDA is characterized as low peripheral circulation perfusion and overloaded pulmonary circulation, accompanying with obvious clinical symptoms, such as tachycardia, systolic murmur and increased non-invasive or invasive respiratory support, etc. Symptomatic PDA is closely related to complications of premature infants and is also

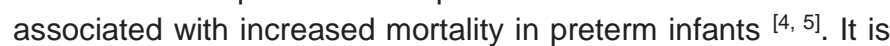

crucial to diagnose and treat in time to avoid side effects brought by excessive treatment. Although many studies have used clinical and echocardiographic parameters to speculate hemodynamic significant PDA, the definition of hsPDA is ongoing debate [6]. From literature reviews, this paper systematically summarizes the identifiable markers of hsPDA in preterm infants, according to non-invasive, biological and clinical approaches.

\section{NON-INVASIVE MARKERS}

\section{Echocardiographic Markers}

The Doppler echocardiography is a gold standard for the diagnosis of PDA, which can be applied to evaluate the effect of PDA on the circulation of premature infants and to monitor the therapeutic effect of PDA ${ }^{[7]}$. Previously, ductal diameter $>1.5 \mathrm{~mm}$ combining with LA/Ao ratio $>1.4: 1$ was used to determine hsPDA by clinicians, as beyond these cutoff, peripheral hypoperfusion and higher rate of ductal misclassification occurs ${ }^{[8-10]}$. Recently, researchers found the LA/AO index can be used to evaluate 
volume load, not PDA. As the value of $L A / A O$ may increase due to volume overload or dysfunctional left ventricular [11]. Based on this concept, some scholars have proposed some new indicators. In 2008, Sonia El-Saiedi showed there is no difference between hemodynamic significant PDA group and hemodynamic insignificant PDA group in LA:AO, but left ventricular Septal Systolic Velocity can be reliably used to detect hsPDA with high sensitivity (100\%) and specificity (94\%). A cut off criterion is $10 \mathrm{~cm} / \mathrm{sec}^{[12]}$. Tugcin Bora Polat found that ductal length from aortic to pulmonary insertion $<5.2 \mathrm{~mm}$ (sensitivity $82 \%$, Specificity $83 \%$ ) and ductal length from ductal constriction to pulmonary insertion $<1.7 \mathrm{~mm}$ (sensitivity $83 \%$ and specificity $86 \%$ ) can be defined as markers for hsPDA [13]. In addition, David W. Jantzen discovered in 2010 that atrial volumes have a linear relationship with body surface area in preterm neonates. Indexed LA volume are prospective markers for hemodynamic significance of PDA [14]. In terms of increased pulmonary circulation, increased left ventricular output ${ }^{[15]}$ and end-diastolic velocity of left pulmonary artery greater than $20 \mathrm{~cm} / \mathrm{s}$ are also described as indicators of hsPDA [11]. As for the peripheral hypoperfusion, researchers found retrograde declining aorta flow associated with the PDA diameter greater than $1.5 \mathrm{~mm}$ may be the best indicator of a hsPDA [16]. Moreover, the declining peripheral artery flow, like cerebral artery and renal artery, can be detected by echocardiography in preterm infant with hsPDA $[17,18]$, so low organ blood flow noted may be an auxiliary approach to identify hsPDA. To summarize the concepts from above researches, the indicators of echocardiographic evaluation usually related to following three aspects: the size of PDA, the situation of pulmonary overcirculation and systemic hypoperfusion. Actually, most researchers try to understand and draw up a clear picture of the hsPDA from ultrasound, they did a gradually improvement on hsPDA diagnosing rely on these 3 directions, and the controversy of medical treatment in hsPDA can be last for decades, which can lead to the changes of echocardiograph performance. More accuracy image and detection from color doppler should be collected in the future.

\section{Other noninvasive markers}

Near-infrared Spectroscopy (NIRS) is a non-invasive technology that can measure regional tissue oxygen saturation level at the bedside [19, 20]. The lower saturation response to peripheral hypoperfusion indirectly, which support the phenomena of systemic steal due to a hsPDA. NIRS can quantitatively estimate the significance of PDA beside echocardiogram (ECHO). A study of 47 babies born less than 29 weeks' gestation were investigated by Valerie Y. Chock and other researchers. They finally confirmed low renal saturation $<66 \%$ was associated with hsPDA in preterm infants with sensitivity of $81 \%$ and specificity of $77 \%{ }^{[20]}$.

Other more convenient and faster detection methods have been studied to determine the prognostic markers of hsPDA. Perfusion Index (PI) is detected by pulse oximeter to indicate the pulse strength. The perfusion of post-ductal is compromised in preterm infants with hsPDA. Kalyan Chakravarthy Balla hypothesized that delta-PI $(\triangle \mathrm{PI}$; pre-ductal-post-ductal $\mathrm{PI})$ is a meaningful clue of symptomatic PDA. They investigated 27 preterm infants $<34$ weeks old, who were divided into two
groups-hsPDA group and no hsPDA group. There is significant difference between two groups on day1 and day3. The study showed $\triangle \mathrm{Pl}>0.85$ on Day 1 could identify hsPDA with sensitivity of $80 \%$ and specificity of $94 \%$. Similarly, $\triangle \mathrm{PI}>0.95$ on Day 3 has the sensitivity and specificity of $80 \%$ and $88.2 \%$, respectively. The available measurements $\Delta \mathrm{PI}$ may help in the assessment of hsPDA ${ }^{[21]}$.

Moreover, mean blood pressure as a common test is also involved to predict a clinically significant PDA requiring treatment [15]. We recommend the more precise relationship between these available paraments and hsPDA should be studied more widely.

\section{Biomarkers}

Pro-B-type natriuretic peptide (proBNP) is synthesized and secreted from the ventricles respond to volume and pressure overload and increased wall tension. In the myocytes, the precursor pro-BNP is cleaved to the biologically active form BNP and the inactive NT-proBNP fragment ${ }^{[22,23]}$. Recently, more and more researchers focused on the relationship between BNP/NTProBNP and hsPDA. A Korean research found BNP is a useful predictor for subsequent hsPDA in ELBW infants at the age of

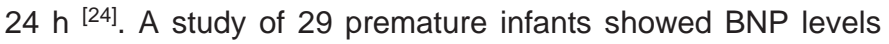
were significantly higher in hsPDA group compared to those without. With a cut-off value of $70 \mathrm{pg} / \mathrm{ml}$, the sensitivity is $92.9 \%$ and the specificity is $73.3 \%{ }^{[25]}$. Another research involving 66 preterm infants discovered the best cutoff BNP concentration for detecting hsPDA was $1110 \mathrm{pg} / \mathrm{ml}$ (sensitivity 100\%, specificity 95.3\%) [26]. While, Jameii Khosroshahi A found the best cutoff point was $450 \mathrm{pg} / \mathrm{ml}$ with the sensitivity and specificity of $92 \%$ and $87 \%$, respectively ${ }^{[27]}$. The varied data is mainly due to different selecting samples, so a large number of samples are needed to obtain more accurate conclusion. Although different dates were existent, all those researches indicating BNP can be used to predict hsPDA.

NT-proBNP, as an inactive fragment, also attract the attention of scientists. NT-proBNP level peaked during the first few days after birth and decreased rapidly within a week. The high level of plasma NT-proBNP during early postnatal period is also a good marker for hsPDA in preterm infants ${ }^{[28,29] .}$

In addition, other biomarkers were also included, such as plasma superoxide dismutase (SOD), ischemia modified albumin (IMA), platelet distribution width (PDW) and absolute nucleated red blood cell(aNRBC). The low SOD activity at 24 to $48 \mathrm{~h}$ after birth is important for identifying infants at risk of symptomatic PDA and the status of SOD may have negative relationship with the incidence of patent ductus arteriosus [30]. IMA is a sort of human plasma protein altered by hypoxia and acidosis, which has lower binding capacity of transitional metal compared with original form [31]. Hasan Kahveci concluded in 2016 IMA is an identifiable marker of hsPDA and can be used to detect therapeutic effects of hsPDA ${ }^{[32]}$. Alona Bin-Nun reported that PDW with the value of $11.45 \mathrm{fL}$ is a good prognostic marker for hsPDA with $65 \%$ sensitivity and $66 \%$ specificity ${ }^{[33]}$ and Alona Bin-Nun showed the cutoff level of aNRBC is $3750 / \mathrm{mm} 3$ for predicting hsPDA(sensitivity $57 \%$, specificity $88 \%$ ) ${ }^{[34]}$. The results above are all based on small samples. Due to the 
boundedness of these studies, more comprehensive data and biomarkers need to be further explored.

\section{Clinical markers}

Clinical features are more readily and rapidly available than others. All examinations mentioned above need opening of the incubator with obvious disturbing and stressing for extremely low birth weight infants. While, clinical markers are more moderate to obtain. Annemarie Kindler established that new four parameters (pulsations of the precordium, bounding femoral pulse, metabolic acidosis and pulmonary deterioration) could assist to diagnose hsPDA and to detect which group immature neonates need PDA-treatment. One point can be scored when one parameter was determined. The cut-off level at score of 2 was optimal with the sensitivity and specificity $84 \%$ and $80 \%$, respectively [35]. In addition, some clinical features such as increased respiratory support, pulmonary edema and feeding intolerance should be markers to highly suspect hsPDA [11]. The clinical features mentioned above combined with corresponding gestational age below should be highly vigilant. Preterm infants with gestational age $\leq 25 w k s$ are at high risk of hsPDA, and gestational age between 26-28wks and >28wks at moderate, low risk, respectively [11]. The chronological age-based organ injury (the IVH at day1-2 after birth, pulmonary hemorrhage at day1-3 and BPD at day1-14) should be in consideration of hsPDA as well ${ }^{[11]}$.

Moreover, the factors from mother should not be ignored. Researchers from the university of Texas Medical Branch hospital in Galveston proposed a hypothesis that antenatal magnesium sulfate exposure may have positive relationship with the incidence of hsPDA in preterm infants as its mechanism to inhibit calcium influx, which is important for closure of ductus arteriosus. As a result, they found that magnesium sulfate exposure did not increase the incidence of hsPDA. Based on partial statistic findings there was a significant negative correlation between antenatal $\mathrm{MgSO}_{4}$ exposure and hsPDA, they speculated that antenatal $\mathrm{MgSO}_{4}$ exposure may play a role of helping with ductal closure [36]. Besides, Elena Fridman discovered maternal aspirin treatment may be associated with a decrease likelihood of hsPDA in preterm infant and may increase infant responsiveness to postnatal medical PDAtreatment [37]. Although attention has been drawn to the relationship between maternal factors and hsPDA recently, further researches should be conducted.

\section{CONCLUSION}

Echocardiographic markers detected mainly involve the size of PDA, pulmonary overcirculation and systematic hypoperfusion, which are crucial to identify hsPDA. Furthermore, the assessment of hsPDA is not determined by a single indicator, but requires multiple perspectives. So other non-invasive, biological and clinical index should be bound considerately. Hemodynamic significant patent ductus arteriosus is closely associated with comorbidity (neonatal necrotizing enterocolitis, intracranial hemorrhage, bronchopulmonary dysplasia, and poor nervous system development) in preterm infants. It is essential to diagnose and treat promptly for premature neonates. To determine the hsPDA is a principal target in clinical area. Further meaningful work needs to be conducted to select the most optimal markers to identify hemodynamic significant patent ductus arteriosus.

\section{REFERENCES}

1. McNamara PJ, Sehgal A. Towards rational management of the patent ductus arteriosus: the need for disease staging. Arch Dis Child Fetal Neonatal Ed, 2007; 92(6):F424-7.

2. Benitz WE. Patent Ductus Arteriosus in Preterm Infants. Pediatrics, 2016; 137(1).

3. Reller M.D., et al. Hemodynamically significant PDA: an echocardiographic and clinical assessment of incidence, natural history, and outcome in very low birth weight infants maintained in negative fluid balance. Pediatr Cardiol, 1985; 6(1):17-23.

4. El-Saiedi S, et al. Development of tissue Doppler-derived predictors of hemodynamically significant patent ductus arteriosus and the ability to incorporate it in targeted neonatal echocardiography protocol. J Saudi Heart Assoc, 2019; 31(2):6974.

5. Lee JA. Practice for preterm patent ductus arteriosus; focusing on the hemodynamic significance and the impact on the neonatal outcomes. Korean J Pediatr, 2019; 62(7):245-251.

6. Zonnenberg I, K de Waal. The definition of a haemodynamic significant duct in randomized controlled trials: a systematic literature review. Acta Paediatr, 2012; 101(3):247-51.

7. de Boode WP. et al. Role of neonatologist-performed echocardiography in the assessment and management of patent ductus arteriosus physiology in the newborn. Semin Fetal Neonatal Med, 2018; 23(4):292-297.

8. Evans N. Current controversies in the diagnosis and treatment of patent ductus arteriosus in preterm infants. Adv Neonatal Care, 2003; 3(4):168-77.

9. Kluckow M, Evans N. Early echocardiographic prediction of symptomatic patent ductus arteriosus in preterm infants undergoing mechanical ventilation. J Pediatr, 1995; 127(5):774-9.

10. Johnson GL. et al. Echocardiographic characteristics of premature infants with patient ductus arteriosus. Pediatrics, 1983; 72(6):86471.

11. Shepherd JL, S Noori. What is a hemodynamically significant PDA in preterm infants? Congenit Heart Dis, 2019; 14(1):21-26.

12. El-Saiedi S. et al., Development of tissue Doppler-derived predictors of hemodynamically significant patent ductus arteriosus and the ability to incorporate it in targeted neonatal echocardiography protocol. J Saudi Heart Assoc, 2019; 31(2):6974.

13. Polat TB, IH Celik, O Erdeve, Early predictive echocardiographic features of hemodynamically significant patent ductus arteriosus in preterm VLBW infants. Pediatr Int, 2016; 58(7):p.589-94.

14. Jantzen DW, et al. Is combined atrial volumetrics by twodimensional echocardiography a suitable measure for quantitative assessment of the hemodynamic significance of patent ductus arteriosus in neonates and infants? Echocardiography, 2010; 27(6):696-701.

15. Katheria $\mathrm{V}$, et al., Early prediction of a significant patent ductus arteriosus in infants $<32$ weeks gestational age. J Neonatal Perinatal Med, 2018; 11(3):265-271.

16. Jain A, PS Shah, Diagnosis, Evaluation, and Management of Patent Ductus Arteriosus in Preterm Neonates. JAMA Pediatr, 2015; 169(9):863-72.

17. Perlman JM, Hill A, Volpe JJ. The effect of patent ductus arteriosus on flow velocity in the anterior cerebral arteries: ductal steal in the premature newborn infant. J Pediatr, 1981; 99(5):767-71.

18. Shimada S, et al., Effects of patent ductus arteriosus on left 
ventricular output and organ blood flows in preterm infants with respiratory distress syndrome treated with surfactant. J Pediatr, 1994; 125(2):270-7.

19. Smith A, AF El-Khuffash, Defining "Haemodynamic Significance" of the Patent Ductus Arteriosus: Do We Have All the Answers? Neonatology, 2020; 117(2): 225-232.

20. Chock VY, et al., Near-infrared spectroscopy for detection of a significant patent ductus arteriosus. Pediatr Res, 2016; 80(5):675680.

21. Balla KC, et al., Perfusion Index-Bedside Diagnosis of Hemodynamically Significant Patent Ductus Arteriosus. J Trop Pediatr, 2016; 62(4):263-8.

22. Maeda $\mathrm{K}$, et al., Plasma brain natriuretic peptide as a biochemical marker of high left ventricular end-diastolic pressure in patients with symptomatic left ventricular dysfunction. Am Heart J, 1998; 135(5 Pt 1):825-32.

23. Weisz DE, PJ McNamara, A El-Khuffash, Cardiac biomarkers and haemodynamically significant patent ductus arteriosus in preterm infants. Early Hum Dev, 2017; 105:41-47.

24. Lee $\mathrm{JH}$, et al., Can early B-type natriuretic peptide assays predict symptomatic patent ductus arteriosus in extremely low birth weight infants? Neonatology, 2013; 103(2):118-22.

25. Sanjeev $\mathrm{S}$, et al. Role of plasma B-type natriuretic peptide in screening for hemodynamically significant patent ductus arteriosus in preterm neonates. J Perinatol, 2005; 25(11):709-13.

26. Choi BM, et al. Utility of rapid B-type natriuretic peptide assay for diagnosis of symptomatic patent ductus arteriosus in preterm infants. Pediatrics, 2005. 115(3): e255-61.

27. Jameii KA, et al. Usefulness of serum brain natriuretic Peptide level for screening hemodynamically significant patent ductus arteriosus in preterm neonates. Iran J Pediatr, 2014; 24(6):766-9.

28. Alenazi SA, N-terminal pro-brain natriuretic peptide measurements in hemodynamically significant patent ductus arteriosus in preterm infants. Pak J Med Sci, 2016; 32(3):580-4.

29. Farombi-Oghuvbu I, et al., N-terminal pro-B-type natriuretic peptide: a measure of significant patent ductus arteriosus. Arch Dis Child Fetal Neonatal Ed, 2008; 93(4):F257-60.

30. Inayat $\mathrm{M}$, et al., Antioxidants and Biomarkers of Oxidative Stress in Preterm Infants with Symptomatic Patent Ductus Arteriosus. Am J Perinatol, 2015; 32(9):895-904.

31. Sadler PJ, A. Tucker and J.H. Viles, Involvement of a lysine residue in the $\mathrm{N}$-terminal $\mathrm{Ni2}+$ and $\mathrm{Cu} 2+$ binding site of serum albumins. Comparison with Co2+, Cd2+ and Al3+. Eur J Biochem, 1994; 220(1):193-200.

32. Kahveci $\mathrm{H}$, et al., Relationship Between Hemodynamically Significant Ductus Arteriosus and Ischemia-Modified Albumin in Premature Infants. Indian J Clin Biochem, 2016; 31(2):231-6.

33. Demirel $\mathrm{G}$, et al., Is high platelet distribution width in the first hours of life can predict hemodynamically significant patent ductus arteriosus in preterm newborns? J Matern Fetal Neonatal Med, 2020; 33(12):2049-2053.

34. Bin-Nun A, et al., Elevated Nucleated Red Blood Cells at Birth Predict Hemodynamically Significant Patent Ductus Arteriosus. J Pediatr, 2016; 177:313-315.

35. Kindler A, et al., Development of a Diagnostic Clinical Score for Hemodynamically Significant Patent Ductus Arteriosus. Front Pediatr, 2017; 5:280.

36. Qasim A, SK Jain, AM Aly, Antenatal Magnesium Sulfate Exposure and Hemodynamically Significant Patent Ductus Arteriosus in Premature Infants. AJP Rep, 2019; 9(4):e353-e356.

37. Fridman E, et al., Effects of maternal aspirin treatment on hemodynamically significant patent ductus arteriosus in preterm infants - pilot study. J Matern Fetal Neonatal Med, 2020: p. 1-6. 